\title{
FREE VIBRATION AND BENDING ANALYSES OF CANTILEVER MICROTUBULES BASED ON NONLOCAL CONTINUUM MODEL
}

\author{
Ömer Civalek, Çiğdem Demir and Bekir Akgöz \\ Akdeniz University, Civil Engineering Department, Antalya-TURKIYE \\ civalek@yahoo.com
}

\begin{abstract}
The equations of motion and bending of Euler-Bernoulli beam are formulated using the nonlocal elasticity theory for cantilever microtubules (MTs). The method of differential quadrature (DQ) has been used for numerical modeling. The size effect is taken into consideration using the Eringen's non-local elasticity theory. Frequencies and deflections of MTs are obtained. Numerical results are presented to show the effect of small-scale effect on bending and vibration of MTs.
\end{abstract}

Key Words: Microtubules, Nonlocal elasticity theory, Vibration, Deflection.

\section{INTRODUCTION}

Microtubules (MTs), microfilaments and intermediate filaments are the main components of cytoskeleton. MTs are proteins organized in a network that is interconnected with microfilaments and intermediate filaments to form the cytoskeleton structures [1]. The mechanical properties of microtubules play an important role in processes such as cell division and intracellular transport [2]. There have been a number of experimental and mathematical studies in the last few decades dealing with the mechanical properties of MTs [3]. Microtubules are the most rigid ones of the cytoskeletal filaments and have the most complex structure. Bending, vibration and buckling analyses of microtubules have been recently investigated using the continuum model [3-6]. Much attention has been devoted also to the mechanical behavior of micro/nano structures such as nanobeams, nanorods and nanotubes [7-15]. In this study, the governing equations for the beam model for cantilever MTs are derived for vibration and bending analysis. The results for static loading and vibration are obtained by using the DQ method.

\section{NONLOCAL CONTINUUM MECHANICS FOR BEAMS}

In the classical elasticity, the stress state of any body at a point $x$ is related to the strain state at the same point $x$. Namely, the constitutive equations of classical (macroscopic) elasticity are algebraic relationships between the stress and strain components. But this theory does not conflict the atomic theory of lattice dynamics and experimental observation of phonon dispersion. As stated by Eringen [16] the linear theory of nonlocal elasticity leads to a set of integropartial differential equations for the displacements field for homogeneous, isotropic bodies. According to the nonlocal elasticity theory of Eringen, the stress at any reference point in the body depends not only on the strains at this point but also on strains at all points of the body. This 
definition of the Eringen's nonlocal elasticity is based on the atomic theory of lattice dynamics and some experimental observations on phonon dispersion. In this theory, the fundamental equations involve spatial integrals which represent weighted averages of the contributions of related strain tensor at the related point in the body. Thus the theory introduces the small length scale effect through a spatial integral constitutive relation. For homogenous and isotropic elastic solids, the linear theory of nonlocal elasticity is described by the following equations [16]:

$$
\begin{gathered}
\sigma_{k l, l}+\rho\left(f_{l}-\frac{\partial^{2} u_{l}}{\partial t^{2}}\right)=0, \\
\sigma_{k l}(x)=\int_{V} \alpha\left(\left|x-x^{\prime}\right|, \chi\right) \tau_{k l}\left(x^{\prime}\right) d V\left(x^{\prime}\right), \\
\tau_{k l}\left(x^{\prime}\right)=\lambda \varepsilon_{m m}\left(x^{\prime}\right) \delta_{k l}+2 \mu \varepsilon_{k l}\left(x^{\prime}\right), \\
\varepsilon_{k l}\left(x^{\prime}\right)=\frac{1}{2}\left(\frac{\partial u_{k}\left(x^{\prime}\right)}{\partial x_{l}^{\prime}}+\frac{\partial u_{l}\left(x^{\prime}\right)}{\partial x_{k}^{\prime}}\right),
\end{gathered}
$$

where $\sigma_{k l}$ is the nonlocal stress tensor, $\rho$ is the mass density of the body, $f_{l}$ is the body (or applied) force density, $u_{l}$ is the displacement vector at a reference point $x$ in the body, $\tau_{k l}\left(x^{\prime}\right)$ is the classical (Cauchy) or local stress tensor at any point $x^{\prime}$ in the body, $\varepsilon_{k l}\left(x^{\prime}\right)$ is the linear strain tensor at point $x^{\prime}$ in the body, $t$ denotes the time, $V$ is the volume occupied by the elastic body, $\alpha\left|x-x^{\prime}\right|$ is the distance in Euclidean form, $\lambda$ and $\mu$ are the Lame constants. The non-local kernel $\alpha\left|x-x^{\prime}\right|$ is defined as the impact of the strain at the point $x^{\prime}$ on the stress at the point $x$ in the elastic body. The value of $\chi$ depends on the ratio $\left(e_{0} a / l\right)$ which is material constant. The value $a$ depends on the internal (granular distance, lattice parameter, distance between $\mathrm{C}-\mathrm{C}$ bonds as molecular diameters) and external characteristic lengths (crack length or wave length) and $e_{0}$ is a constant appropriate to each material for adjusting the model to match reliable results by experiments or some other theories. If $\alpha|x|$ takes on a Green function of a linear differential operator given as [16]

$$
\mathfrak{R} \alpha\left(\left|x^{\prime}-x\right|\right)=\delta\left(\left|x^{\prime}-x\right|\right)
$$

the nonlocal constitutive relation given by Eq.(2) is reduced to the differential equation

$$
\mathfrak{R} \sigma_{k l}=\tau_{k l}
$$


Furthermore the integro-partial differential equation given by Eq. (1) is also reduced to the following partial differential equation

$$
\tau_{k l, l}+\Re\left(f_{l}-\rho \ddot{u}_{k}\right)=0
$$

Eringen (3) proposed a nonlocal model for this linear differential operator given as

$$
\mathfrak{R}=1-\left(e_{0} a\right)^{2} \nabla^{2}=0
$$

where $\nabla^{2}$ is the Laplacian. Consequently, the constitutive relations can be written as

$$
\left[1-\left(e_{0} a\right)^{2} \nabla^{2}\right] \sigma_{k l}=\tau_{k l}
$$

\section{GOVERNING EQUATIONS FOR MTs}

It is accepted that some mechanical behavior such as vibration, bending and buckling of the beam-like micro structures based on nonlocal elasticity theory are entirely different from their counterparts based on the classical (macro) beam theory [2-10]. Thus the theory based on size dependent nonlocal elasticity theory could serve as a more reasonable and proper approach in mechanical modeling of micro and nano sized components of nano mechanical devices [11-23]. The nonlocal theory of elasticity proposed by Eringen [16] has been widely used in the past five years in many nano mechanical problems including dislocation, crack, wave propagation, vibration analysis of nanobeams, nanotubes, carbon nanotubes, and microtubules. For MTs in one dimensional case, the nonlocal constitutive relations can be written as below [6]

$$
\sigma_{x x}-\left(e_{0} a\right)^{2} \frac{\partial^{2} \sigma_{x x}}{\partial x^{2}}=E \varepsilon_{x x}
$$

where $\sigma_{x x}$ is the axial stress, $\varepsilon_{x x}$ is the axial strain, E is the Young modulus. Assume that the transverse displacement of beam along y axial axis is $w(x, t)$ in terms of spatial coordinate $x$ and time variable $t$. For transverse vibration of MTs, the equilibrium conditions of Euler-Bernoulli beam can be written as

$$
\frac{\partial^{2} M(x, t)}{\partial x^{2}}=\rho A \frac{\partial^{2} w(x, t)}{\partial t^{2}}
$$

According to the linear theory of Euler-Bernoulli beam, the strain-displacements and the moment are given by

$$
\varepsilon=-y \frac{\partial^{2} w(x, t)}{\partial x^{2}}, \quad M(x, t)=\int_{A} y \sigma d A
$$


In this stage, multiplying by $y$ on both sides of Eq. (12) and integrating over the crosssection area of the beam, we obtain

$$
\int_{A} \sigma_{x x} y d A-\left(e_{0} a\right)^{2} \int_{A} y \frac{\partial^{2} \sigma}{\partial x^{2}} d A-\int_{A} E y \varepsilon d A=0
$$

After some mathematical manipulations, we have

$$
M(x, t)-\left(e_{0} a\right)^{2} \frac{\partial^{2} M(x, t)}{\partial x^{2}}+E I \frac{\partial^{2} w(x, t)}{\partial x^{2}}=0
$$

By differentiating this equation with respect to the variable $x$ twice, we obtain

$$
\frac{\partial^{2} M(x, t)}{\partial x^{2}}-\left(e_{0} a\right)^{2} \frac{\partial^{4} M(x, t)}{\partial x^{4}}+E I \frac{\partial^{4} w(x, t)}{\partial x^{4}}=0
$$

Finally, by substituting Eq. (11) into Eq. (16) we obtain the following governing nonlocal equation for vibration of MTs based on Euler-Bernoulli beam theory [6]

$$
E I \frac{\partial^{4} w(x, t)}{\partial x^{4}}+\rho A \frac{\partial^{2} w(x, t)}{\partial t^{2}}-\left(e_{0} a\right)^{2} \rho A \frac{\partial^{4} w(x, t)}{\partial x^{2} \partial t^{2}}=0
$$

It is exactly seen from the Eq. (17) that the local Euler-Bernoulli beam theory is obtained when the parameter $e_{0}$ is set identically to zero. In this case, nonlocal bending moment by using a harmonic function for $w(\mathrm{x})$ can be written as

$$
M(x)=\frac{E I}{L^{2}}\left[-\frac{\partial^{2} w(x)}{\partial x^{2}}-\left(e_{0} a\right)^{2} \rho A \frac{L^{2}}{E I} \omega^{2} w(x)\right]
$$

where $\omega$ is the angular frequency. If we consider the Euler-Bernoulli beam subjected to a distributed load, the general nonlocal equation for this case is given below

$$
E I \frac{d^{4} w(x)}{d x^{4}}+q(x)\left(e_{0} a\right)^{2} \frac{d^{2} q(x)}{d x^{2}}=q(x)
$$

by using the relation between the load and deflection, that is

$$
E I \frac{\partial^{4} w(x)}{\partial x^{4}}=q(x)
$$

Substituting the Eq. (20) into Eq. (19) we obtain 


$$
E I \frac{d^{4} w(x)}{d x^{4}}+q(x)\left(e_{0} a\right)^{2} \frac{d^{6} w(x)}{d x^{6}}=q(x)
$$

Clamped-free boundary conditions are considered. These are:

For clamped (C) end (at $\mathrm{x}=0$ )

For free $(\mathrm{F})$ end (at $\mathrm{x}=\mathrm{L})$

$$
w=0 \text { and } d w / d x=0
$$

$$
V=0 \text { and } M=0
$$

\section{DIFFERENTIAL QUADRATURE (DQ) METHOD}

Differential quadrature (DQ) method is a relatively new numerical technique in applied mechanics. The method of DQ can yield accurate solutions with relatively fewer grid points. It has been also successfully employed for different solid and fluid mechanics problems [17-21]. Unlike the DQ that uses the polynomial functions, such as power functions, Lagrange interpolated, and Legendre polynomials as the test functions, harmonic differential quadrature method uses harmonic or trigonometric functions as the test functions. Shu and Xue [17] proposed an explicit means of obtaining the weighting coefficients for the harmonic DQ. $f(x)$ is approximated by a Fourier series expansion in the form

$$
f(x)=c_{0}+\sum_{k=1}^{N / 2}\left(c_{k} \cos \frac{k \pi x}{L}+d_{k} \sin \frac{k \pi x}{L}\right)
$$

and the Lagrange interpolated trigonometric polynomials are taken as

$$
h_{k}(x)=\frac{\sin \frac{\left(x-x_{0}\right) \pi}{2} \cdots \sin \frac{\left(x-x_{k}\right) \pi}{2} \sin \frac{\left(x-x_{k+1}\right) \pi}{2} \cdots \sin \frac{\left(x-x_{N}\right) \pi}{2}}{\sin \frac{\left(x_{k}-x_{0}\right) \pi}{2} \cdots \sin \frac{\left(x_{k}-x_{k-1}\right) \pi}{2} \sin \frac{\left(x_{k}-x_{k+1}\right) \pi}{2} \cdots \sin \frac{\left(x_{k}-x_{N}\right) \pi}{2}}
$$

for $k=0,1,2, \ldots, N$. According to the HDQ, the weighting coefficients of the first-order derivatives $A_{i j}$ for $i \neq j$ can be obtained by using the following formula:

$$
A_{i j}=\frac{(\pi / 2) P\left(x_{i}\right)}{P\left(x_{j}\right) \sin \left[\left(x_{i}-x_{j}\right) / 2\right] \pi} ; i, j=1,2,3, \ldots, N,
$$

where 


$$
P\left(x_{i}\right)=\prod_{j=1, j \neq i}^{N} \sin \left(\frac{x_{i}-x_{j}}{2} \pi\right) ; \text { for } \mathrm{j}=1,2,3, \ldots, N
$$

The weighting coefficients of the second-order derivatives $B_{i j}$ for $i \neq j$ can be obtained using following formula:

$$
B_{i j}=A_{i j}\left[2 A_{i i}^{(1)}-\pi c t g\left(\frac{x_{i}-x_{j}}{2}\right) \pi\right] ; \quad i, j=1,2,3, \ldots, N,
$$

The weighting coefficients of the first-order and second-order derivatives $A_{i j}^{(p)}$ for $i=j$ are given as

$$
A_{i l}^{(p)}=-\sum_{j=1, j \neq i}^{N} A_{l j}^{(p)} ; p=1 \text { or } 2 ; \text { and for } i=1,2, \ldots, N
$$

By using DQ discretization the Eq. (21) takes the form for bending

$$
\frac{E I}{L^{4}} \sum_{j=1}^{N} D_{i j} w_{j}+q_{i}\left(e_{0} a\right)^{2} \sum_{j=1}^{N} F_{i j} w_{j}=q_{i}
$$

For vibration, the discrete form of governing equation takes the form:

$$
E I \sum_{j=1}^{N} D_{i j} W_{j}-\omega^{2} \rho A W_{i}+\left(e_{0} a\right)^{2} \rho A \omega^{2} \sum_{j=1}^{N} B_{i j} W_{j}=0
$$

The weighting coefficient of the fourth- and sixth-order derivatives $\left(D_{\mathrm{ij}}, F_{\mathrm{ij}}\right)$ can be computed easily from $B_{i j}$ by

$$
D_{i j}=\sum_{k=1}^{N} B_{i k} B_{k j} ; F_{i j}=\sum_{k=1}^{N} D_{i k} B_{k j}
$$

\section{NUMERICAL RESULTS}

In this section, several examples of MTs having clamped-free boundary conditions are examined. For this purpose, some numerical results in the forms of graphs are presented using the method of DQ. The material and geometric constants of MTs are given in Table 1 . The symbol C-F, represents the MT having clamped edge at $x=0$ and free edge at $x=L$. To determine the accuracy and convergence of the present method for deflection analysis of MTs, numerical experimentation was carried out by varying the number of grid points $N$. 
Table 1. Material and geometric values of MTs

\begin{tabular}{cc}
\hline Parameter & Value \\
\hline $\mathrm{E}$ & $2 \times 10^{9} \mathrm{~N} / \mathrm{m}^{2}$ \\
\hline $\mathrm{I}$ & $105 \times 10^{-34} \mathrm{~m}^{4}$ \\
\hline$\rho$ & $1470 \mathrm{~kg} / \mathrm{m}^{3}$ \\
\hline$v$ & 0.3 \\
\hline
\end{tabular}

The obtained results of non-dimensional static deflections and vibration computed for different $N$ values are shown in Table 2. Exact analytical solution [4,5] is also given for comparison. It is important to state that the results given by these references [4] related to carbon nanotubes based on beam theory. However, we solved microtubules problem firstly. So we compare only coefficient (the results are presented as nondimensional).

Table 2. Comparison of non-dimensional maximum deflection $\left(w \times E I / q \mathrm{~L}^{4}\right)$ of C-F MTs under uniformly distributed loading

\begin{tabular}{|c|c|c|c|c|c|c|}
\hline \multirow{2}{*}{$\mathrm{e}_{0} \mathrm{a} / \mathrm{L}$} & $\begin{array}{c}\text { Analytical } \\
\text { Result } \\
\text { Ref.4 }\end{array}$ & \multicolumn{5}{|c|}{ Present DQ solutions } \\
\cline { 5 - 7 } & $\mathrm{N}=5$ & $\mathrm{~N}=7$ & $\mathrm{~N}=9$ & $\mathrm{~N}=11$ & $\mathrm{~N}=13$ \\
\hline 0.0 & 0.1250 & 0.12518 & 0.12504 & 0.12501 & 0.12501 & 0.12501 \\
\hline 0.20 & 0.0250 & 0.02511 & 0.02507 & 0.02506 & 0.02506 & 0.02506 \\
\hline
\end{tabular}

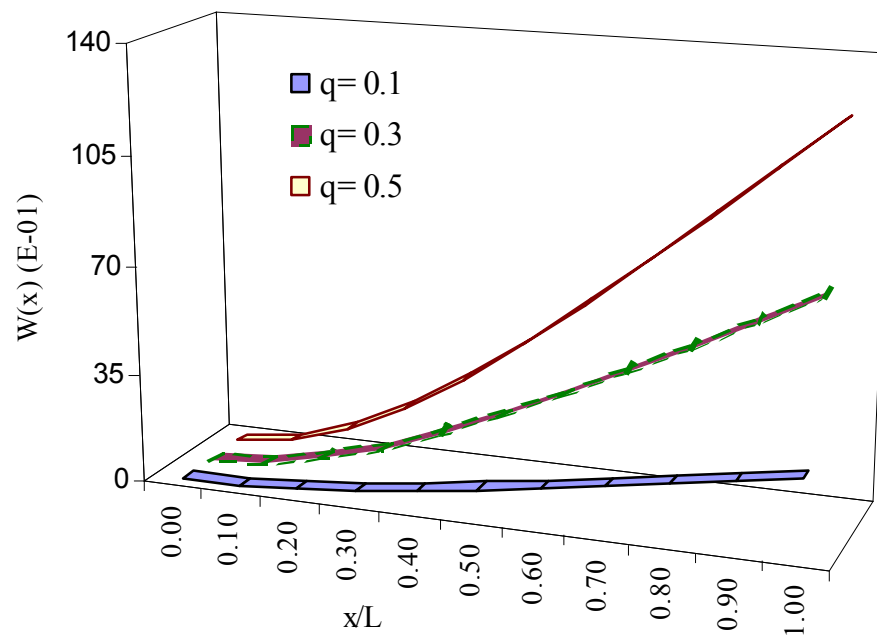

Fig. 1. Deflection of C-F Carbon nanotubes $\left(\mathrm{e}_{0} \mathrm{a}=0.3 \times 10^{-9} \mathrm{~m}\right)$

Excellent agreement has been achieved between the present results and the results obtained by analytical formula given by Reddy and Pang [4]. It is seen from this table 
that when the grid point numbers reaches $N=7$ the present method gives accurate predictions for the deflections. For frequency values, however, there are no results for comparison. Figs. 1 and 2 show the displacement along the length of MTs for different values of load and nonlocal parameters. It can be seen that the effect of nonlocal parameter on the deflection is insignificant. In general, the nonlocal parameter results in an increase in the transverse deflection of MTs under uniformly distributed load.

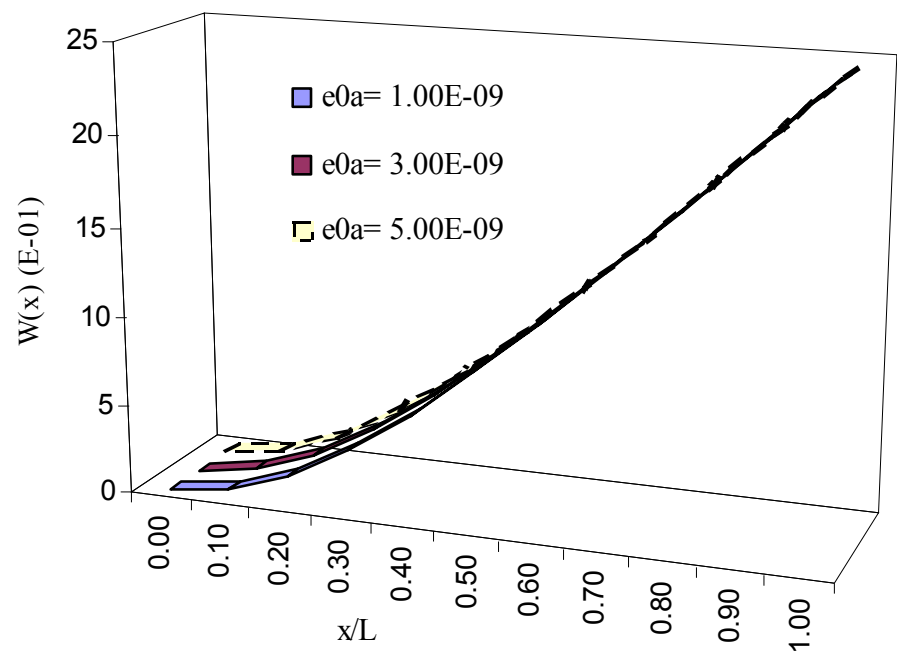

Fig. 2. Deflection of C-F microtubules for different nonlocal parameter $(q=0.2 \mathrm{~N} / \mathrm{m})$

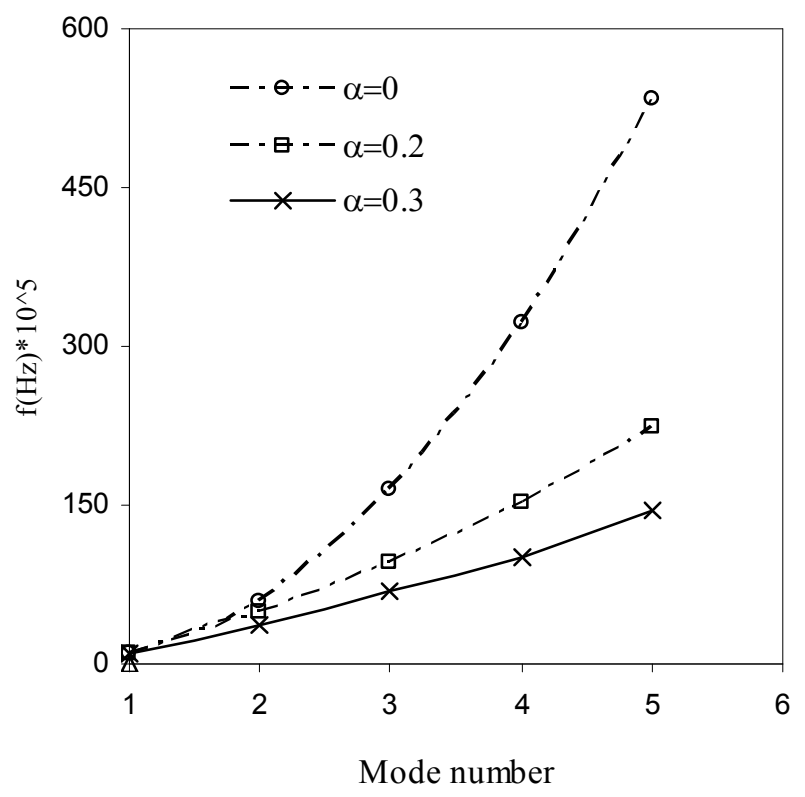

Fig.3. Frequencies of C-F MTs for different nonlocal parameter $\left(L=5 \times 10^{-6} \mathrm{~m} ; \alpha=\mathrm{e}_{0} \mathrm{a} / \mathrm{L}\right)$ 
Fig. 3 shows the variation of the frequency value with mode number of C-F microtubules for different nonlocal parameter. It is seen that the frequency values for all the three nonlocal parameters $(\alpha=\mathrm{e} 0 \mathrm{a} / \mathrm{L})$ increase as mode number increases. It is also shown that, the frequency values decrease with increasing nonlocal parameter.

\section{CONCLUDING REMARKS}

In this paper, free vibration and static behavior of MTs are investigated. The numerical results show that the nonlocal parameter is affected on static and dynamic behaviour of MTs. The method is suitable for the problem considered due to its generality, simplicity, and potential for further development. Although not provided here, the method is also useful in providing buckling solutions of MTs using nonlocal elasticity theory.

\section{Acknowledgements}

The financial support of the Scientific Research Projects Unit of Akdeniz University is gratefully acknowledged.

\section{REFERENCES}

1. J. Howard, Mechanics of Motor Proteins and the Cytoskeleton. Sinauer Associates, Inc, Sunderland, MA.,2001

2. D. Boal, Mechanics of the cell, Cambridge University Press, Cambridge, 2002.

3. C.Y. Wang, C.Q. Ru, A. Mioduchowski, Orthotropic elastic shell model for buckling of microtubules. Phys Rev. E, 74,052901, 2006.

4. J.N. Reddy, S.D. Pang, Nonlocal continuum theories of beams for the analysis of carbon nanotubes. J Appl Phy, 103,023511, 2008.

5. J.N. Reddy, Nonlocal theories for bending, buckling and vibration of beams. Int $J$ Eng Sciences 45,288-307, 2007.

6. K.M. Liew, Q. Wang, Analysis of wave propagation in carbon nanotubes via elastic shell theories, International Journal of Engineering Science, 45, 227-241, 2007.

7. Q. Wang, K.M. Liew, Application of nonlocal continuum mechanics to static analysis of micro- and nano-structures. Physics Letters A, 363,236-242, 2007.

8. Ö. Civalek, Ç. Demir, B. Akgöz, Static Analysis of Single Walled Carbon Nanotubes (SWCNT) Based On Eringen's Nonlocal Elasticity Theory. International Journal of Engineering and Applied Sciences (IJEAS), 1(2) 47-56,2009.

9. Ç. Demir, Ö. Civalek, B. Akgöz, Free Vibration Analysis of Carbon Nanotubes Based On Shear Deformable Beam Theory By Discrete Singular Convolution Technique. Mathematical and Computational Applications, In press, 2009.

10. C. Li, C.Q. Ru, A. Mioduchowski, Torsion of the central pair microtubules in eukaryotic flagella due bending-driven lateral buckling, Biochemical and Biophysical Research Communications 351,159-164, 2006. 
11. C.Y. Wang, L.C. Zhang, Circumferential vibration of microtubules with long axial wavelength, Journal of Biomechanics 41,1892-1896, 2008.

12. X.S. Qian, J.Q. Zhang, C.Q., Ru, Wave propagation of orthotropic microtubules, J Appl Phys 101, 084702-084717, 2007.

13. B. Akgöz, Modeling of Carbon nanotubes as Timoshenko and Euler beams, B.Sc. Thesis, (in Turkish), Akdeniz University,2009.

14. Ç. Demir, B. Akgöz, Ö. Civalek, Free Vibration And Bending Analysis Of Carbon Nanotubes Using Nonlocal Euler Beam Theory, International Symposium on Engineering and Architectural Sciences of Balkan, Caucasus and Turkic Republics, 2009.

15. Ç. Demir, Ö. Civalek, A. Korkmaz, Frequencies Analysis Of Tapered Carbon Nanotubes Embedded In Two-Parameter Elastic Foundation, International Symposium on Engineering and Architectural Sciences of Balkan, Caucasus And Turkic Republics, 2009.

16. A.C. Eringen, On differential equations of nonlocal elasticity and solutions of screw dislocation and surface waves. J. Applied Physics 54,4703-4710, 1983.

17. C. Shu, H. Xue, Explicit computations of weighting coefficients in the harmonic differential quadrature. J. Sound Vib. 204(3)549-555, 1997.

18. Ö. Civalek, Application of Differential Quadrature (DQ) and Harmonic Differential Quadrature (HDQ) For Buckling Analysis of Thin Isotropic Plates and Elastic Columns. Engineering Structures, 26(2)171-186, 2004.

19. Ö. Civalek, M. Ülker, Harmonic Differential Quadrature (HDQ) For Axisymmetric Bending Analysis Of Thin Isotropic Circular Plates. Int. J. Structural Engineering and Mechanics, 17(1)1-14, 2004.

20. C. H. W. Ng, Y. B. Zhao, Y. Xiang and G. W. Wei, On the Accuracy and Stability of a Variety of Differential Quadrature Formulations for the Vibration Analysis of Beams. International Journal of Engineering and Applied Sciences (IJEAS),1(4) 1-25, 2009.

21. M. Şimşek, Static Analysis of a Functionally Graded Beam under a Uniformly Distributed Load by Ritz Method, International Journal of Engineering and Applied Sciences (IJEAS),1(3) 1-11, 2009.

22. M. Aydoğdu, Axial vibration of nanorods with the nonlocal continuum rod model. Physica E 41,861-864, 2009.

23. R. Artan, A. Tepe, The initial values method for buckling nonlocal bars with application in nanotechnology. European Journal of Mechanics A/Solids 27,469-477, 2008. 